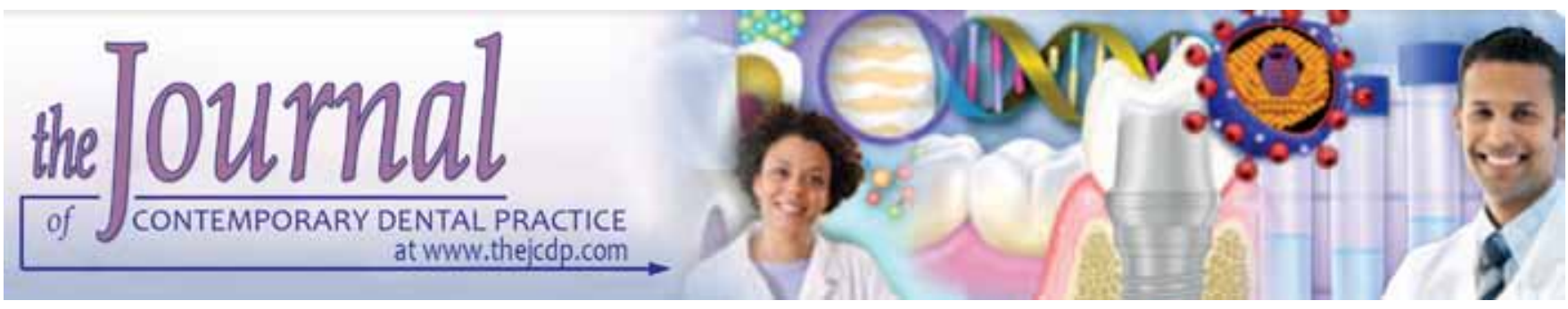

\title{
Developmental Malformation of Primary and Permanent Dentition: A Rare Sequel of Trauma
}

\section{Garima Jindal, Dipanshu Kumar}

\section{ABSTRACT}

Dentofacial injuries that occur prior to the eruption of primary teeth can result in developmental disturbances not only in the primary but also in permanent dentition. Here we report a rare case of long term sequelae of trauma in a female child of 4 to 5 months of age which resulted in dilaceration and impaction of maxillary primary central incisors and subsequent enamel hypoplasia of the permanent maxillary central incisors.

Keywords: Impaction, Dilaceration, Enamel hypoplasia.

How to cite this article: Jindal G, Kumar D. Developmental Malformation of Primary and Permanent Dentition: A Rare Sequel of Trauma. J Contemp Dent Pract 2013;14(5):944-947.

Source of support: Nil

Conflict of interest: None declared

\section{BACKGROUND}

Dentofacial trauma occuring prior to the eruption of primary teeth can not only result in developmental disturbance and failure of eruption of primary dentition but rarely may have a late sequel on permanent dentition. Andreason reported the incidence of dental injuries to be commonest among all facial injuries and account for $18 \%$ of all somatic injuries seen in the oral region in children aged 0 to 6 years old. ${ }^{1}$

Tooth impaction refers to failure of tooth to erupt due to mechanical blocking and the tooth thus remains unerupted beyond the normal time of eruption. ${ }^{2}$ An impacted tooth is a phenomenon usually associated with permanent dentition and is rarely seen during the development of primary dentition (reported prevalence ratio of $1: 10,000^{3}$ ). The impaction in primary dentition may be primary, meaning that the teeth have never erupted due to some physical barrier in the eruption pathways (also known as primary failure of eruption) or it may be secondary impaction, due to ankylosis, the teeth after eruption are reimpacted. ${ }^{3}$ Primary tooth impactions can occur because of various reasons which include hard tissue abnormalities like odontomes, soft tissue conditions such as myxofibrous hyperplasia and ameloblastic fibroma and malpositioning of the tooth germ, either due to trauma or unknown reasons. ${ }^{3}$ The etiology of primary tooth impaction could involve a local traumatic event, that might not only result in dilaceration of the primary tooth but also lead to subsequent disturbances in the developing permanent dentition. Dilaceration is a term used to describe a distortion occurring during development of the tooth which disrupts the normal axial relationship between the crown and the root. It occurs most commonly in permanent incisors as a result of trauma to primary predecessors. ${ }^{4}$

The highly unusual condition of impaction of primary anterior tooth associated with dilaceration has not been commonly reported in the dental literature. The two cases reported in the literature involved impactions due to abnormal positioning of the primary tooth germs, with no history of trauma. We report a rare case of long term sequel of trauma at 4 to 5 months of age in a female child resulting in dilaceration and impaction of maxillary primary central incisors and subsequent enamel hypoplasia of the permanent maxillary central incisors.

\section{CASE REPORT}

A 3-year-old girl was referred to the Department of Pediatric and Preventive Dentistry with the chief complaint of missing maxillary primary central incisors and parents reported that these primary teeth had never erupted. The medical history of the child revealed that the child was born by unassisted vaginal delivery at 40 weeks. There was no perinatal stress and the child required no special care. Neonatal laryngoscopy and endotracheal intubation were not used nor the child was born with natal or neonatal teeth, excluding these as the possible causes of missing teeth. Child had undertaken normal vaccination programme and there was no history of significant childhood illness.

On anamnesis, parents recalled an episode of facial trauma at 4 to 5 months of age wherein child fell onto her face and was hit by the corner of the bed causing 


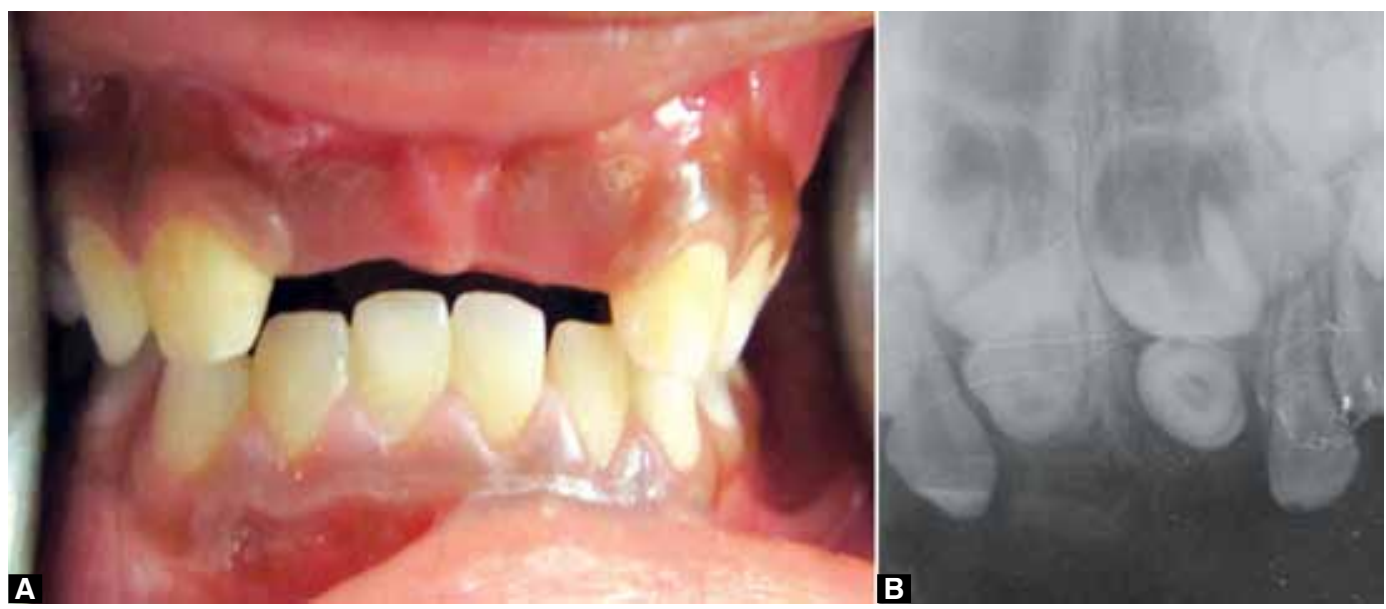

Figs 1A and B: (A) Frontal view of the patient at 3 years of age. (B) Intraoral periapical radiograph showing horizontally impacted maxillary primary central incisors

bruising of her upper lip and laceration of labial aspect of anterior maxillary ridge. The facial injury was managed conservatively, requiring no stitches and was treated by a physician. The wound healed uneventfully in few days. No dental consultation was done at the time of injury.

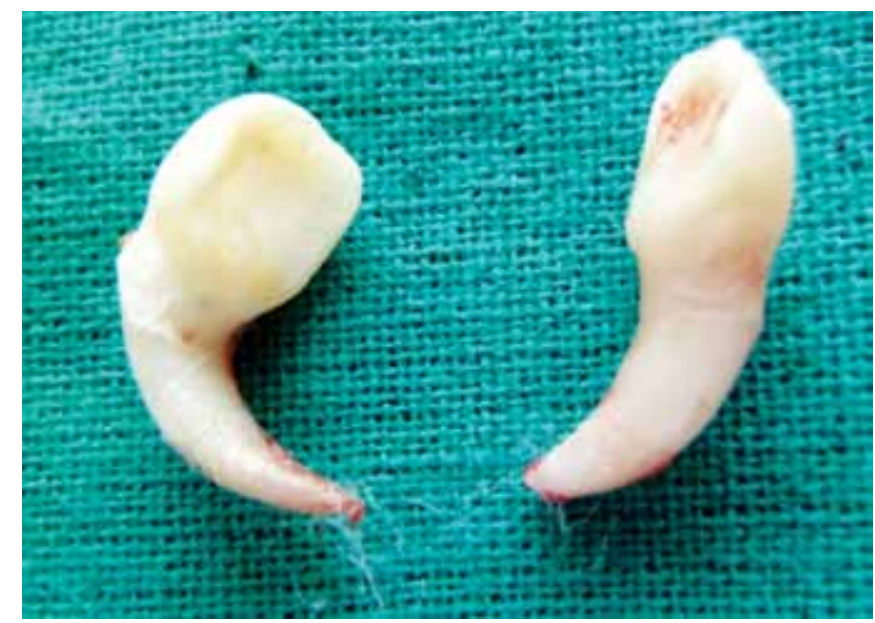

Fig. 2: Extracted primary maxillary central incisors depicting dilacerated roots
Intraoral examination of the patient revealed that maxillary primary central incisors were missing (Fig. 1A). A mild bulge was visible in the region of missing incisors but the teeth could not be palpated. The remaining primary dentition was present in normal shape and alignment. No abnormality was noted in gingiva and alveolar bone.

On intraoral periapical radiograph, the maxillary primary central incisors appeared to be horizontally impacted. (Fig. 1B) and the developing permanent central incisors were closely related to the impacted teeth. In addition it appeared that there were possible abnormalities in the crowns of developing permanent central incisors.

Considering the age of the patient, position of the primary incisors and location of the permanent central incisors, it was decided to surgically extract the primary central incisors under general anesthesia. After raising the mucoperiosteal flap, the crowns were exposed and were found to be lying buccopalatally. The primary incisors were carefully extracted taking care not to disturb the permanent crowns. The teeth were dilacerated with a sharp bend at the cervical one-third

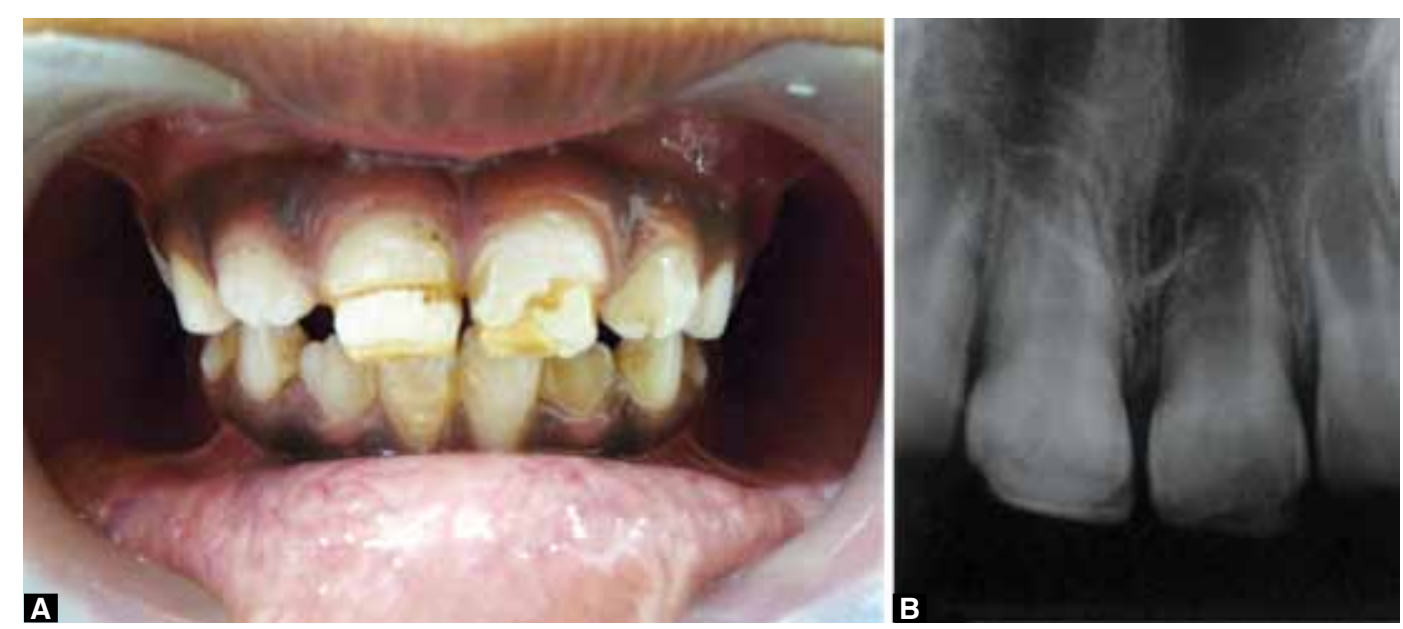

Figs 3A and B: (A) Frontal view of the patient at 8 years of age. (B) Intraoral periapical radiograph showing hypoplastic permanent maxillary central incisors 
of the root creating its vestibular angulation (Fig. 2). At a review visit, the healing progressed satisfactorily and periodic recall visits were advised to monitor the developing dentition.

Five years after the procedure the patient returned to our department with the chief complaint of discolored permanent maxillary central incisors. Intraoral examination revealed the incisal one-third of the maxillary central incisors to be hypoplastic with brownish discoloration (Fig. 3A) and a narrow horizontal groove was visible encircling the crowns cervical to the discolored area. Radiographic examination revealed a radiolucent area in the incisal third and transverse radiolucent line at the level of indentation of the crowns. (Fig. 3B). Patient was advised laminate veneers for esthetic improvement of upper permanent central incisors but the patient did not undergo further treatment because of economic reasons.

\section{DISCUSSION}

Pindborg stated failure of eruption of a primary tooth to be a rare phenomenon. ${ }^{5}$ There are only two previously reported cases of primary tooth impaction associated with dilaceration found in the literature. Bimstein et $\mathrm{al}^{6}$ reported a case of root dilaceration and stunting of two unerupted mandibular incisors, while Kinirons ${ }^{7}$ reported a similar case of a maxillary incisor that had failed to erupt and was subsequently found to be dilacerated. In both these cases authors postulated the cause of tooth impaction to be developmental in origin. However, the most common cause of dilaceration has been reported to be a traumatic event during the development of tooth. ${ }^{4}$ The factors responsible for impaction of permanent teeth do not necessarily account for primary teeth impaction, as they occupy superficial position within the developed alveolar arches. ${ }^{8}$ Impaction is common in teeth with malformation confined to either the crown or root. ${ }^{1}$

In the present case, it might be postulated that the traumatic event at 4 to 5 months of age may have caused disruption of the dental follicle in the early stages of development of maxillary primary central incisors. Primary incisors erupt between 6 and 9 months after birth and their root development is complete around 12 to 18 months later. ${ }^{4}$ To affect the cervical one-third of the root of a primary incisor, as in this case, the trauma must have occurred soon after crown formation was complete. At the time of occurrence of this event (4-5 months of age), the root formation of the primary maxillary central incisors and calcification of the crown of permanent maxillary central incisors would have just begun. Vestibular root angulation of the primary central incisors could have occurred because the crown in the dental follicle was displaced by the traumatic event but its continued root development had to conform to the associated hard structure. These events during the primary tooth development perhaps resulted in dilaceration and subsequent noneruption of the primary incisors. It has also been well documented that intraoral endotracheal intubation in infants can produce enough pressure on the developing alveolus to cause disturbances of developing tooth germs. ${ }^{9}$ Thus, blunt trauma in a 4 to 5 months old infant could be the etiology behind follicular displacement with subsequent dilaceration and impaction of primary incisor tooth. In the present case, extraction was planned because normal eruption of the dilacerated teeth was not likely to occur and to prevent interference with the development and eruption of succedaneous teeth.

This case also presents a rare complication of dilacerated impacted primary central incisors resulting in the enamel hypoplasia of the permanent maxillary incisors. Hard tissue formation of maxillary permanent incisors begins at 3 to 4 months after birth and trauma during this period might result in anomalies of the developing permanent maxillary central incisors. ${ }^{9}$ Injuries occuring during the initial stages of tooth development may interfere with normal enamel formation due to disturbance in different stages in ameloblastic development, i.e. the morphogenic, organizing, formative and maturation stage. ${ }^{10}$ Experiments in monkeys have shown that circular enamel hypoplasia represents localized damage to the ameloblasts in their formative stages due to traumatic displacement of already formed hard tissue in relation to the developing soft tissue. ${ }^{10}$ This would correlate with the crown abnormalities of the upper permanent maxillary central incisors found in the present case.

\section{CONCLUSION}

Most injuries to the primary dentition occur between the ages of 2 and 4 years, by when primary incisors are fully formed and their root apices are in close approximation with the permanent successors. ${ }^{1}$ Damage to the developing permanent tooth germ would be easily sustained if the primary root comes into contact with it. ${ }^{1}$ However, in the present case, injury occurred before the eruption of primary dentition (4-5 months) and it can be safely assumed that the displaced developing primary tooth might have traumatized the tissue adjacent to the permanent tooth germ and possibly the odontogenic epithelium, thereby interfering with its enamel mineralization.

\section{CLINICAL SIGNIFICANCE}

Hence, trauma incurred even in early infancy may manifest its sequelae not only in the primary but also in the permanent 
dentition. Thus, complete evaluation of the full extent of early childhood injuries must await complete development of all the involved permanent teeth. This case report emphasizes the need of providing adequate and thorough information to parents pertaining to the sequelae of dentoalveolar trauma in early infancy and the importance of regular review of both the primary as well as permanent dentitions.

\section{REFERENCES}

1. Flores MT, Malmgren B, Andersson L, et al. Guidelines for the management of traumatic dental injuries. III. Primary teeth. Dent Traumatol 2007 Aug; 23(4):196-202.

2. Otsuka Y, Mitomi T, Tomizawa M, Noda T. A review of clinical features in 13 cases of impacted primary teeth. Int J Paediatr Dent 2001;11(1):57-63.

3. Kapur A, Goyal A, Jaffri S. Management of inverted impacted primary incisors: an unusual case. J Indian Soc Pedod Prev Dent 2008;26(1):26-28.

4. Kilpatrick NM, Hardman PJ, Welbury RR. Dilaceration of a primary tooth. Int J Paediatr Dent 1991;1(3):151-153.

5. Pindborg JJ. Pathology of the dental hard tissues. WB Saunders Co: Philadelphia. 1970;p.41.
6. Bimstein E. Root dilaceration and stunting in two unerupted primary incisors. ASDC J Dent Child 1978;45(3):223-225.

7. Kinirons MJ. Dilaceration and failure to erupt of nonsuccessional teeth. Journal of Irish Dental Association 1983; 29:53-54.

8. Adams TW. An impacted primary lateral incisor as a cause of delayed eruption of a permanent totth: case report. Pead Dent. 1998;20:121-123.

9. Cole B, Welbury R. Malformation in the primary and permanent dentitions following trauma prior to tooth eruption: a case report. Endod Dent Traumatol 1999;15:294-296.

10. Andreasen JO, Ravn JJ. Enamel changes in the permanent teeth after trauma to their primary predecessors. Scan J Dent Res 1973; 81(3):203-209.

\section{ABOUT THE AUTHORS}

\section{Garima Jindal (Corresponding Author)}

Senior Resident, Department of Pedodontics, King George Medical College (erstwhile CSSMU) Lucknow, Uttar Pradesh, India e-mail: dr.gjindal@gmail.com

\section{Dipanshu Kumar}

Senior Resident, Department of Pedodontics, King George Medical College (erstwhile CSSMU) Lucknow, Uttar Pradesh, India 